\title{
Cost-Effective Fiber Multiplexing System Based on Low Coherence Interferometers and Application to Temperature Measurement
}

\author{
Meng JIANG ${ }^{*}$, Zhongze ZHAO, Kun LI, Zeming WANG, Yage ZHAN, \\ Hongying ZHOU, and Fu YANG
}

College of Science Donghua University of China, Shanghai, 201620, China

*Corresponding author: Meng JIANG_Ｅ-mail: jiangmeng@dhu.edu.cn

\begin{abstract}
Based on the low-coherence interferometric principles, a cost-effective all-fiber Mach-Zehnder multiplexing system is proposed and demonstrated. The system consists of two interferometers: sensing interferometer and demodulation interferometer. By scanning an optical tunable delay line back and forth constantly with a stable speed, sensing fibers with different optical paths can be temporal interrogated. The system is experimentally proved to have a high performance with a good stability and low system noises. The multiplexing capacity of the system is also investigated. An experiment of measuring the surrounding temperature is carried out. A sensitivity of $12 \mu \mathrm{m} /{ }^{\circ} \mathrm{C}$ is achieved within the range of $20^{\circ} \mathrm{C}$ to $80{ }^{\circ} \mathrm{C}$. This low cost fiber multiplexing system has a potential application in the remote monitoring of temperature and strain in building structures, such as bridges and towers.
\end{abstract}

Keywords: Mach-Zehnder interferometer; low-coherence; multiplexing fiber system

Citation: Meng JIANG, Zhongze ZHAO, Kun LI, Zeming WANG, Yage ZHAN, Hongying ZHOU, et al., "Cost-Effective Fiber Multiplexing System Based on Low Coherence Interferometers and Application to Temperature Measurement," Photonic Sensors, 2016, 6(4): 318-323.

\section{Introduction}

Fiber optical sensors have been widely researched in the fields of physics, chemistry, bio-medicine, and industry [1-5] in recent decades because of their high performance compared with the traditional electronic sensors. With the advantages of anti-corrosion and anti-electromagnetic interference, a variety of new fiber optical sensors appeared in the world's laboratories [6-12]. Optical fiber interferometry has been wildly used to produce optical sensors with extremely high resolution [13, 14]. Low coherence interferometry (LCI), white light interferometry, has been widely developed as a multiplexing sensing system with high sensitivity, large measurement range, high resolution, and low cost. One of the medical applications of LCI is optical coherence tomography (OCT) [15], in which it has been proved that the axial resolution of OCT improves with the bandwidth. The LCI technique can also be used to simultaneously resolve the mode profile and to measure the intermodal dispersion of guided modes of a few-mode fiber [16]. The interference cross-correlation function is only significant over an optical path difference approximating to the coherence length of the light source. The zero-order interference fringe can be identified for absolute position of optical path

Received: 19 May 2016 / Revised: 31 August 2016

(C) The Author(s) 2016. This article is published with open access at Springerlink.com

DOI: $10.1007 / \mathrm{s} 13320-016-0344-6$

Article type: Regular 
difference. The interferogram is obtained by a temporal scan of the group delay in one arm of the demodulation interferometer. By measuring the phase delay in one of the sensing interferometer's arms, the corresponding change in the interference signal can be obtained, and the measurand can be deduced. Most importantly, LCI is a multiplexing system with high capacity and consequent demodulation mechanism [9-11]. LCI can integrate multiplex sensors onto one single optical signal without requiring the use of other complex time or frequency multiplexing techniques. The sensing interferometer which can transfer sensing signal to phase delay can be multiplexed and interrogated by the LCI system.

In this paper, we demonstrate a cost-effective all-fiber multiplexing sensing scheme based on LCI [12], which measures the absolute optical path difference between reference fiber and sensing fiber. The interrogation of the system is realized by scanning an optical tunable delay line with a stable speed. This multiplexing system provides high performance with a good stability and low system noises. The multiplexing capacity is also investigated here. An experiment of measuring the surrounding temperature is carried out. A sensitivity of $12 \mu \mathrm{m} /{ }^{\circ} \mathrm{C}$ is achieved within the range of $20{ }^{\circ} \mathrm{C}$ to $80{ }^{\circ} \mathrm{C}$ by our system.

\section{Experimental principles}

Low-coherence measurement method has been elaborated in classical optics [17]. It utilizes broad-band light source (low-coherence light source) such as a laser diode (LD) or a light-emitting diode (LED), combined with Mach-Zehnder, Michelson or other interferometer, to achieve a high precision measurement.

\subsection{Low-coherence interferometer}

Low coherence interferometry is simply formed by two interferometers: sensing interferometer and demodulation interfereometer. The structure of lowcoherence interferometer based on Mach-Zehnder interferometer is shown in Fig. 1.

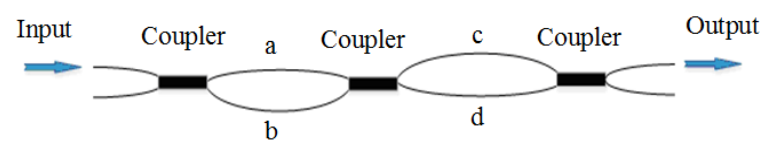

Fig. 1 Structure of low-coherence Mach-Zehnder interferometer

Light input is divided into two beams equally by a $2 \times 2$ fiber coupler and propagates through "a" and "b" arms (sensing arm and reference arm), respectively. The electric components of the two beams can be expressed as follows [18]:

$$
\begin{gathered}
E_{\mathrm{a}}(\omega t, \varphi)=A_{\mathrm{a}}(\omega \tau) \exp (\mathrm{i} \varphi) \\
E_{\mathrm{b}}(\omega t, \varphi)=A_{\mathrm{b}}(\omega \tau) \exp \left[\mathrm{i}\left(\varphi+k \delta_{1}\right)\right]
\end{gathered}
$$

where $A$ is the electric amplitude, $\varphi$ is the phase angle, $\delta_{1}$ is the optical path difference (OPD) between "a" and " $b$ " arms, $\kappa$ is the wave vector, and $i$ is the imaginary part. After the two beams are coupled into "c" and "d" arms, there will be four beams:

$$
\begin{gathered}
E_{\mathrm{ad}}=A_{\mathrm{ad}} \exp (\mathrm{i} \varphi) \\
E_{\mathrm{bd}}=A_{\mathrm{bd}} \exp \left[\mathrm{i}\left(\varphi+k \delta_{1}\right)\right] \\
E_{\mathrm{ac}}=A_{\mathrm{ac}} \exp \left[\mathrm{i}\left(\varphi+k \delta_{2}\right)\right] \\
E_{\mathrm{bc}}=A_{\mathrm{bc}} \exp \left[\mathrm{i}\left(\varphi+k\left(\delta_{1}+\delta_{2}\right)\right)\right]
\end{gathered}
$$

where $\delta_{2}$ is the OPD introduced between "c" and "d" arms. Once the OPD between $E_{\mathrm{bd}}$ and $E_{\mathrm{ac}} \Delta \delta=$ $\left|\delta_{2}-\delta_{1}\right|$ is less than the coherence length of the light source, the interference fringes can be obtained at the output of the fiber.

In our experimental setup, the OPD $\delta_{1}$ will be influenced by the under test parameters. The OPD $\delta_{2}$ is scanning to compensate the OPD $\delta_{1}$. In our system, a motorized optical tunable delay line (OTDL, General Photonics, MDL-002) is embedded in the fiber "d" arm to adjust the $\delta_{2}$.

\subsection{Optical fiber characteristics for temperature}

The group delay of the optical pulse propagating in the optical fiber can be represented by [19]

$$
\tau=\frac{L N}{c}
$$

where $L$ is the length of optical fiber, $N$ is the effective refractive-index of the fiber core, and $c$ is the velocity of light in vacuum. 
Hence, the fiber temperature delay drift constant is defined by

$$
K_{f}=\frac{d \tau}{d T} \frac{1}{L}=\frac{1}{C}\left(\frac{d N}{d T}+\frac{N}{L} \frac{d L}{d T}\right) .
$$

Substituting the thermal expansion coefficient $\left(\approx 5.5 \times 10^{-7} /{ }^{\circ} \mathrm{C}\right)$ and the thermo-optical coefficient $\left(\approx 1 \times 10^{-5} /{ }^{\circ} \mathrm{C}\right)$ for silica into $(8)$, the $K_{f}$ of fiber is approximately $0.036 \mathrm{ps} /\left(\mathrm{m} \cdot{ }^{\circ} \mathrm{C}\right)$. When surrounding temperature of a certain length of fiber ('a' arm in Fig. 1) changes, the OPD $\delta_{1}$ will be changed relatively. The interference signal will emerge when the OPD $\delta_{2}$ (introduced by the scanning OTDL) can compensate the changed $\delta_{1}$. By observing the position drift of the interference fringes, the temperature variation can be detected.

\section{Experimental setup}

The structure of our multiplexing sensing system is shown in Fig. 2. An amplified spontaneous emission (ASE) light source with the central wavelength of $1567 \mathrm{~nm}$ and spectral bandwidth of $73 \mathrm{~nm}$ is used as a low-coherence light source here. The system is composed of two Mach-Zehnder interferometers: the first one is multi-channel for multiplexing sensors; the second interferometer which contains an OTDL is used to demodulate all sensing signals. The polarization controller in the demodulation interferometer is used to minimize the polarization influence to obtain a clearly interference fringes.

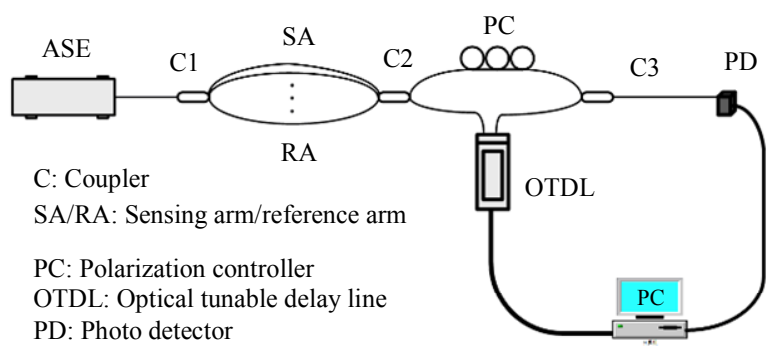

Fig. 2 Structure of the multiplexing sensing system.

The output signal is detected by an InGaAsbased photodetector which is connected by a data acquisition (DAQ) card installed in the computer. The OTDL can scan back and forth within a range of
0 to $330 \mathrm{ps}$, corresponding to an optical path of 0 to $99 \mathrm{~mm}$. In our system setup, the OTDL and DAQ card are computer-controlled by a Labview program. We connect a reference arm and two sensing arms in the experiment for demonstration. The length of reference fiber is set as $1970 \mathrm{~mm}$, while the two sensing arms are $1986 \mathrm{~mm}$ and $1990 \mathrm{~mm}$, respectively.

\subsection{Demodulate scheme}

When the OTDL scans for one single trip, four interference fringes would appear in the entire interferogram, as shown in Fig. 3.

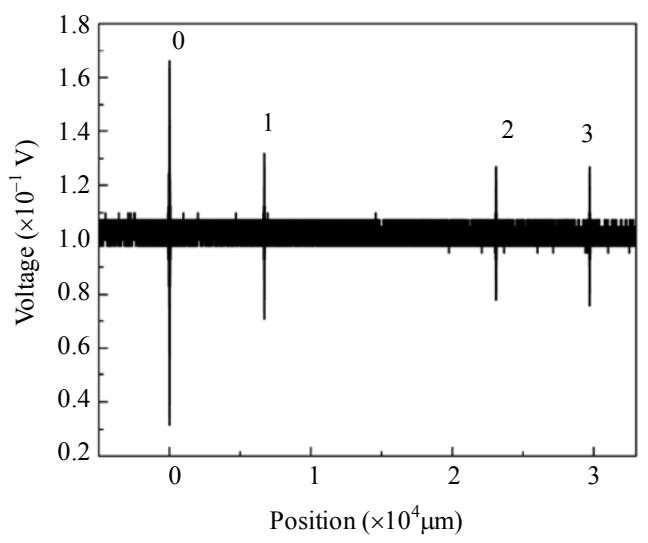

Fig. 3 Measured interferogram of the multiplexing system when it has two fiber sensors (the scanning speed is $16 \mathrm{ps} / \mathrm{s}$ ).

The highest interference fringe is the zero-order interfering where the OPD $\delta_{2}$ approaches zero. The position of this fringe can be the reference for measure " 2 " is generated when the OPD introduced by OTDL can compensate the OPD between the reference arm and first fiber sensing arm, while fringe " 3 " is generated when the OPD between the reference arm and second fiber sensing arm is compensated. Fringe " 1 " is generated when the OPD of two sensing arms is compensated, which could be regarded as a "cross-talk" of the system here.

The OPD introduced by the sensing interferometers should be less than the range of the OTDL, so that the fringes can be obtained during the OTDL scans. We should note that the "cross-talk" fringes (like fringe "1") caused between sensors should be designed away from the sensing fringes. 
Therefore, we set the OPD between the first fiber sensor and reference as half of the OTDL's scanning range. And other fiber sensors have longer optical paths than the first one. In this way, all of the "cross-talk" fringes could appear between the fringe " 0 " and the fringe " 2 ", so that one can distinguish the sensing fringes from "cross-talk" fringes easily.

As illustrated in Fig. 4, by employing a DAQ card and a program written by Labview, the peak position of the zero-order interference fringe envelope can be accurately located. So do the fringes " 2 " and " 3 ". Figure 4 also indicates that the scanning speed of the OTDL is uniform, which is important in extracting the interference positions. Table 1 gives the four-peak points positions of fringes envelope in the interferogram in Fig. 3.

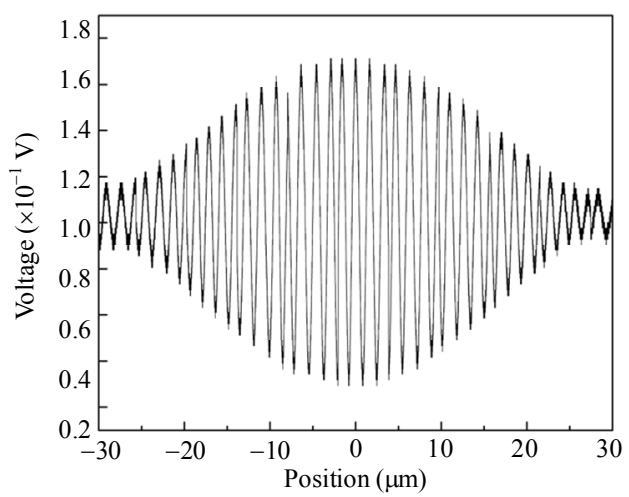

Fig. 4 Detailed fringes of the zero-order interferogram (the scanning speed is $0.01 \mathrm{ps} / \mathrm{s}$ ).

Table 1 Peak positions of interference fringes envelope in Fig. 3.

\begin{tabular}{cc}
\hline Fringe & Peak position $(\mu \mathrm{m})\left(20^{\circ} \mathrm{C}\right)$ \\
\hline 0 & 0 \\
1 & 6703 \\
2 & 22195 \\
3 & 29714 \\
\hline
\end{tabular}

To prove the stability of the system, we carry out the repeatability test on the position of the fringe "2" under certain environment at room temperature $\left(22.2 \pm 0.2{ }^{\circ} \mathrm{C}\right)$. The peak point positions of the fringe "2" are given in Table 2 . The stability of the system is proved to be good. Since the accuracy of the OTDL is $0.01 \mathrm{ps}$, the accuracy of our system is $3 \mu \mathrm{m}$ correspondingly.
Table 2 Peak positions of fringe "2" under stable environment.

\begin{tabular}{ccc}
\hline Number & Temperature $\left({ }^{\circ} \mathrm{C}\right)$ & Peak position $(\mu \mathrm{m})$ \\
\hline 1 & 22.0 & 22199 \\
2 & 22.4 & 22204 \\
3 & 22.3 & 22201 \\
4 & 22.3 & 22201 \\
5 & 22.3 & 22201 \\
6 & 22.3 & 22201 \\
7 & 22.4 & 22204 \\
8 & 22.4 & 22204 \\
9 & 22.4 & 22204 \\
10 & 22.4 & 22204 \\
\hline
\end{tabular}

\subsection{Temperature measurement}

As analyzed in Section 2.2, the system can be utilized to detect the surrounding temperature. Theoretically, parameters which can influence the phase delay of the optical fiber can be detected by our system, such as strain, vibration, and stretching.

In the experiment, a single mode fiber with 0.9-meter length of the first sensing fiber is heated by a temperature oven. The scanning speed of the OTDL is $3 \mu \mathrm{m} / \mathrm{s}$, and the sample rate of the DAQ card is $8000 \mathrm{~Hz}$. The position drift of the peak "2" with the temperature changes is illustrated in Fig. 5. The scanning speed of the OTDL is set as $8 \mathrm{ps} / \mathrm{s}$ so that all the interference fringes could be discerned on the screen of the computer, and it will take about forty seconds to demodulate all sensors. The sensitivity of the temperature is $12 \mu \mathrm{m} /{ }^{\circ} \mathrm{C}$, with the range of $20{ }^{\circ} \mathrm{C}$ to $80{ }^{\circ} \mathrm{C}$. The group delay of $12 \mu \mathrm{m}$ is about $0.04 \mathrm{ps}$ in the OTDL, which corresponds to the optical fiber of $0.03 \mathrm{ps} /\left(\mathrm{m} \cdot{ }^{\circ} \mathrm{C}\right)$. So the measurement results match with the theoretical sensitivity in Section 2.2. Since the accuracy of the system is $3 \mu \mathrm{m}$, the resolution of this experiment is $0.25{ }^{\circ} \mathrm{C}$. One can get higher sensitivity and resolution by simply lengthening the sensing fiber under measured temperature.

The capacity of this multiplexing system heavily relies on the scanning range of the OTDL, which is 0-330 ps in our system. So the OTDL could introduce $0-99 \mathrm{~mm}$ optical path in free space into the Mach-Zehnder interferometer. In the experiment, temperature change of $100{ }^{\circ} \mathrm{C}$ could cause a $1.2-\mathrm{mm}$ 
position shift of the interference fringes. Furthermore, the cross-talks made by the sensor array should be separated from the sensing signals. In our system, the cross-talks are designed close to the zero order interference fringes by means of designing the OPD between every two sensors less than the OPD between the reference optical fiber and the minimal optical sensor:

$$
\mathrm{OPD}_{0,1} \gg \mathrm{OPD}_{0, N}
$$

where " 0 " means the reference arm, and " 1 " and " $N$ " mean the sensors which have the minimal and maximal optical paths.

Therefore, only half of the scanning range of OTDL is used for sensing interference fringes, thus the capacity of our system could be

$$
n \ll(99 / 2) / 1.2 \approx 40
$$

where " $n$ " represents the number of sensors.

The capability of this system can be further enlarged by utilizing a longer scanning range OTDL, which is commercial available. Besides temperature, strain on the fiber sensor can also involve additionally optical phase change in the system. If we package each fiber sensor properly to make it only sensitive to temperature or strain, our system can be used to demodulate all the sensors to measure both temperature and strain.

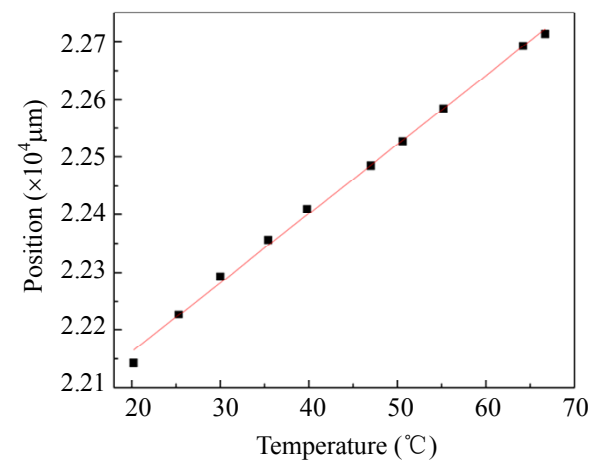

Fig. 5 Position drifts of the interference fringe envelope peak "2" versus temperature.

\section{Conclusions}

In this paper, an optical fiber multiplexing sensing system based on LCI is built up. By scanning an optical tunable delay line back and forth constantly with a stable speed, fiber sensors with different optical paths can be temporal interrogated. This multiplexing system shows high performance with a good stability in the experiment. The "cross-talk" fringes between multiplexed sensors can be designed to be eliminated from the sensing signals. The capacity of the system is also discussed. An experiment of measuring the surrounding temperature is carried out. A sensitivity of $12 \mu \mathrm{m} /{ }^{\circ} \mathrm{C}$ is achieved within the range of $20{ }^{\circ} \mathrm{C}$ to $80{ }^{\circ} \mathrm{C}$ by our system, which matches well with theoretical results.

Our proposed multiplexing sensing system will be useful for the remote monitoring of the temperature and strain. By incorporating fiber optic sensor arrays into structures such as bridges and buildings, smart structures can be realized with relatively low cost.

\section{Acknowledgment}

This work was supported by Pujiang Program (No. 14PJ1400100) and the Fundamental Research Funds for the Central Universities of China (No. 2232014D3-28).

Open Access This article is distributed under the terms of the Creative Commons Attribution 4.0 International License (http://creativecommons.org/licenses/by/4.0/), which permits unrestricted use, distribution, and reproduction in any medium, provided you give appropriate credit to the original author(s) and the source, provide a link to the Creative Commons license, and indicate if changes were made.

\section{References}

[1] N. B. Kosa, "Key issues in selecting plastics optical fibers used in novel medical sensors," Proc. of SPIE, 1991, 1592: 114-121.

[2] T. G. Gaillorenze, "Optical fiber sensor technology," IEEE Journal of Quantum Electronics, 1982, 18(4): 626-665.

[3] R. C. Spooncer, "Fiber optics in instrumentation," in Handbook of Measurement Science, Ed: P. H. Sydenham \& R. Thorn, Chichester. UK: Wiley, 1992: 1691-1720 
[4] H. Chen and Y. Liang, "Analysis of tunable asymmetric fiber F-P cavity for fiber strain sensor edge-filter demodulation," Photonic Sensors, 2014, 4(4): 338-343.

[5] Q. Li and H. Chen, "Design of fiber magnetic field sensor based on fiber Bragg grating Fabry-Perot cavity ring-down spectroscopy," Photonic Sensors, 2015, 5(2): 189-192.

[6] K. T. V. Grattan, "Fiber optic sensors-the way forward," Measurement: Journal of the International Measurement Confederation, 1987, 5: 122-134.

[7] E. M. Dianov, S. A. Vasiliev, A. S. Kurkov, O. I. Medvedkov, and V. N. Protopopov, "In-fiber MachZehnder interferometer based on a pair of long-period gratings," in 22th European Conference on Optical Communication, Oslo, 1996.

[8] D. P. Hand and P. St J. Russell, "Photo induced refractive-index changes in germanosilicate fiber," Optics Letters, 1990, 15(2): 102-104.

[9] J. L. Brooks, R. H. Wentworth, R. C. Youngquist, M. Tur, B. Y. Kim, and H. J. Shaw, "Coherence multiplexing of fiber-optic interferometricsensors," IEEE Journal of Lightwave Technology, 1985, 3(5): 1062-1072.

[10] W. V. Sorin and D. M. Baney, "Multiplexed sensing using optical low-coherence reflectometry," IEEE Photonics Technology Letters, 1995, 7(8): 917-919.

[11] L. B. Yuan and J. Yang, "Two-loop-based low-coherence multiplexing fiber optic sensor network with a Michealson optical path demodulator," Optics Letters, 2005, 30(6): 601-603.
[12] L. Jin, W. G. Zhang, Q. C. Tu, and X. Y. Dong, "Applications of interferential technique in designing optics fiber sensors," Laser and Optoelectronics Exhibition, 2004, 41(7): 54-55.

[13] C. D. Butter and G. B. Hocker, "Fiber optic strain gauge," Applied Optics, 1978, 17(18): 2867-2869.

[14] S. R. Kidd, P. G. Sinha, J. S. Barton, and J. D. C. Jones, "Interferometric fiber sensors for measurement of surface heat transfer rates on turbine blades," Optics and Lasers in Engineering, 1992, 16(2-3): 207-221.

[15] P. Jansz, S. Richardson, G. Wild, and S. Hinckley, "Modeling of low coherence interferometry using broadband multi-Gaussian light sources," Photonic Sensors, 2012, 2(3): 247-258.

[16] Y. Z. Ma, Y. Sych, G. Onishchukov, S. Ramachandran, U. Peschel, B. Schmauss, et al., "Fiber-modes and fiber-anisotropy characterization using low-coherence interferometry," Applied Physics B, 2009, 96(2): 345-353.

[17] M. Born and E. Wolf, "Principles of optics. New York: Pergamon Press, 1986.

[18] K. T. V. Grattan and B. T. Meggitt, Optoelectronics, imaging and sensing series: optical fiber sensor technology volume 2 devices and technology. London: Chapman \& Hall, 1998: 169-171.

[19] X. Z. Hu, Fiber optic and fiber cable. Beijing: Publishing House of Electronics Industry, 2007: 24-243. 